\title{
Moral wounds run deep: exaggerated midbrain functional network connectivity across the default mode network in posttraumatic stress disorder
}

\author{
Braeden A. Terpou, PhD; Chantelle S. Lloyd, PhD, Maria Densmore, BSc; \\ Margaret C. McKinnon, PhD; Jean Théberge, PhD; Richard W. J. Neufeld, PhD; \\ Rakesh Jetly, MD; Ruth A. Lanius, MD, PhD
}

\begin{abstract}
Background: A moral injury occurs when a deeply held moral code has been violated, and it can lead to the development of symptoms of posttraumatic stress disorder (PTSD). However, the neural correlates that differentiate moral injury and PTSD remain largely unknown. Intrinsic connectivity networks such as the default mode network (DMN) appear to be altered in people with PTSD who have experienced moral injury. However, brainstem, midbrain and cerebellar systems are rarely integrated into the intrinsic connectivity networks; this is a critical oversight, because these systems display marked differences in people with PTSD and are thought to underlie strong moral emotions such as shame, guilt and betrayal. Methods: We conducted an independent component analysis on data generated during script-driven memory recall of moral injury in participants with military- or law enforcement-related PTSD ( $n=28)$, participants with civilian-related PTSD $(n=28)$ and healthy controls exposed to a potentially morally injurious event $(n=18)$. We conducted group-wise comparisons of functional network connectivity differences across a DMN-correlated independent component, with a particular focus on brainstem, midbrain and cerebellar systems. Results: We found stronger functional network connectivity in the midbrain periaqueductal grey $\left(t_{71}=4.95, p_{\mathrm{FDR}}=0.028, k=39\right)$ and cerebellar lobule IX $\left(t_{71}=4.44, p_{\mathrm{FDR}}=0.046, k=49\right)$ in participants with civilianrelated PTSD as compared to healthy controls. We also found a trend toward stronger functional network connectivity in the midbrain periaqueductal grey $\left(t_{71}=4.22, p_{\mathrm{FDR}}=0.076, k=60\right)$ in participants with military- or law enforcement-related PTSD as compared to healthy controls. Limitations: The significant clusters were large, but resolution is generally lower for subcortical structures. Conclusion: In PTSD, the DMN appears to be biased toward lower-level, midbrain systems, which may drive toxic shame and related moral emotions that are common in PTSD, highlighting the depth at which moral injuries are represented neurobiologically.
\end{abstract}

\section{Introduction}

In unusually high-stress situations, a person may perpetuate, fail to prevent, or witness an event that contradicts their deeply held moral beliefs and expectations. ${ }^{1}$ When someone does something that goes against their beliefs, it is referred to as an act of commission; when someone fails to do something in line with their beliefs, it is referred to as an act of omission. Acts of both commission and omission are considered to be potentially morally injurious events, as are situations that involve a sense of betrayal on the part of a trusted leader, peer or institution..$^{2-5}$ As their name suggests, potentially morally injurious events may lead to moral injury, which refers to the distressing psychological, behavioural, social and spiritual aftermath caused by such an event. ${ }^{6}$ Similar to a traumatic event, a potentially morally injurious event may precipitate adverse mental health outcomes, including posttraumatic stress disorder (PTSD), depression and suicidality. ${ }^{7-11}$

Most research investigating moral injury has been in members of the military and veterans, given that the nature of war presents situations in which a person may experience an event that contradicts the values they live by in civilian life. Examples of military-related potentially morally injurious events include killing or harming another person, a situation in which a medic cannot attend to everyone who has been harmed, or a situation in which someone freezes or fails to perform an active duty in a hostile setting. ${ }^{12-14}$ Still, although most research investigating moral injury has been with the military, moral injury can occur in a variety of occupations in which traumatic events are frequent. ${ }^{6}$ Research investigating moral injury in health care workers has revealed trends similar to those in the military. Examples of health care-related potentially morally

Correspondence to: R.A. Lanius, Department of Psychiatry, Western University, University Hospital, 339 Windermere Rd., London ON N6A 5A5; ruth.lanius@Ihsc.on.ca

Submitted Jul. 16, 2021; Revised Nov. 22, 2021; Accepted Dec. 5, 2021

Cite as: J Psychiatry Neurosci 2022 February 17;47(1). doi: 10.1503/jpn.210117 
injurious events include making triage decisions with life or death implications, or a situation in which a physician feels that they should have saved a patient's life but could not. ${ }^{15}$

Many of the situations described above would be considered traumatic because they involve an actual or perceived threat of serious injury or loss of life. A great deal of overlap exists between the definitions of traumatic events and potentially morally injurious events. However, not all traumatic events result in a moral injury, and it is possible to experience moral injury and not meet the criteria for PTSD, ${ }^{16}$ suggesting that these constructs are dissociable, at least clinically. PTSD includes symptoms that are not central to moral injury, such as hyperarousal, hypervigilance and dissociation. Moral injury is a much more specific construct, defined largely by the presence of strong moral emotions such as shame, guilt and betrayal. ${ }^{17-19}$ Although shame, guilt and betrayal are often reported in PTSD, they are not part of the diagnostic criteria. Interestingly, such moral emotions have an inherent self-reflective and socialcognitive quality to them, unlike fear, the preeminent emotion in PTSD. ${ }^{7}$ The inability to forgive oneself and engagement in self-sabotaging behaviour have been suggested as central to the construct of moral injury. ${ }^{12,20}$ Such disturbances to self-related processing are thought to be caused by the evolving emotional responses brought on by shame and guilt. ${ }^{8,21}$

Intense feelings of shame, guilt and betrayal lie at the core of moral injury; in addition to the distress these emotions may cause independently, recent research suggests that they may also have a more general effect on adult psychopathology. Because adverse childhood experiences, moral injury and PTSD frequently occur together, Roth and colleagues ${ }^{22}$ sought to examine the role that moral injury plays in the relationship between adverse childhood experiences and mental health symptoms in adulthood. They included a sample of participants who were regularly exposed to potentially traumatic work-related events (i.e., public safety personnel such as police officers, firefighters or paramedics) and revealed that symptoms of moral injury - namely, feelings of shame, guilt and betrayal - mediated the relationship between adverse childhood experiences and symptoms of depression, dissociation and PTSD in adulthood. This was the first study to identify moral injury as a mechanism involved in the relationship between adverse childhood experiences and adult mental health symptoms, highlighting that the cognitions and emotions related to moral injury are critical for evolving psychopathology.

Despite these findings, however, only preliminary research has begun to disentangle the neural correlates that underlie moral injury and PTSD. ${ }^{23}$ Dissociable neural activity has been revealed in the resting state in US veterans, ${ }^{24}$ and during scriptdriven memory recall related to moral injury events in members of the Canadian Armed Forces and law enforcement officers. ${ }^{25}$ During script-driven memory recall, Lloyd and colleague ${ }^{25}$ found stronger activity in regions that underlie defensive responding (e.g., the postcentral gyrus and the innate alarm system), viscerosensory responding (e.g., the posterior insula and the dorsal anterior cingulate cortex) and top-down modulatory control (e.g., the dorsolateral prefrontal cortex) in participants with PTSD as compared to healthy controls exposed to a moral injury event. Moreover, shame scores related to moral injury were anticorrelated with the activity of the superior frontal gyrus - a region in the dorsomedial prefrontal cortex that is involved in self-reflection. The authors suggested that the anticorrelation pattern may speak to a diminished capacity for self-reflection in PTSD, a pattern that likely perpetuates the moral injury. ${ }^{17}$ On balance, the research reviewed here points toward altered activity patterns across brain regions that are involved in social cognition, moral reasoning and emotion regulation during the recollection of a potentially morally injurious event, highlighting the distributed aftermath of moral injuries, especially when they occur alongside PTSD. ${ }^{9}$

Critically, the brain regions described by Lloyd and colleagues ${ }^{25}$ overlap considerably with central hubs involved in the intrinsic connectivity networks, which display altered activity and functional connectivity patterns in PTSD. ${ }^{26-28} \mathrm{Al}-$ though marked differences emerge in the patterns of activity and functional connectivity across the intrinsic connectivity networks, these differences have corticocentric focus and generally fail to incorporate brainstem, midbrain and cerebellar systems into the broader picture. Midbrain systems and the periaqueductal grey in particular display overactivity and altered functional connectivity with the default mode network (DMN) in people with PTSD, ${ }^{29,30}$ and yet these systems are rarely discussed in tandem with the intrinsic connectivity networks, a critical oversight in mental health research. Integrating midbrain systems into the intrinsic connectivity networks may help improve our understanding of complex emotions such as shame, which evokes an urge to cringe, hide or withdraw (lower level), but also has an inherent socialcognitive and self-reflective quality (higher level). ${ }^{31,32}$

Located around the cerebral aqueduct, the periaqueductal grey modulates the autonomic nervous system, which connects the central nervous system to the viscera, suggesting that this structure has a vital role in primary affect (e.g., fear, rage, panic, care, lust, play, seeking) ${ }^{33,34}$ and innately conserved defence responses (e.g., fight, flight, faint, tonic immobility), which rely fundamentally on the autonomic nervous system. ${ }^{35-37}$ In PTSD, altered patterns of activity and functional connectivity have been revealed between the periaqueductal grey and the intrinsic connectivity networks in the resting state, ${ }^{38-42}$ as well as during subliminal, trauma-related stimulus processing. ${ }^{29,30,41,42}$ In particular, Terpou and colleagues ${ }^{29,43}$ have demonstrated stronger functional connectivity directed by the periaqueductal grey toward the medial prefrontal cortex and the precuneus (critical DMN hubs) in participants with PTSD; the authors took this finding to suggest that the periaqueductal grey may be not only involved in the DMN in PTSD, but also driving it.

In the present study, we sought to explore functional network connectivity related to the intrinsic connectivity networks in people with and without PTSD who were exposed to moral injury, with a particular focus on the brainstem, midbrain and cerebellar systems and whether they are recruited differently in PTSD. We employed a script-driven memory recall task related to a moral injury event — a DMN-mediated task — to address these study aims. We used a data-driven, computational approach (i.e., independent component analysis [ICA]) to identify the intrinsic connectivity networks. In line with Terpou and colleagues, ${ }^{43}$ we hypothesized that the midbrain and the 
periaqueductal grey in particular would be recruited more strongly across the DMN in participants with PTSD as compared to healthy controls. Any such pattern of enhanced midbrain functional network connectivity across the DMN could help to explain why many participants with PTSD report persistent viscerosensory hyperactivity, especially when they are engaged in memory recall and self-reflection, functions mediated by the DMN. ${ }^{44,45}$ More broadly, this study aimed to highlight the important but underinvestigated role of the brainstem, midbrain and cerebellar systems in relation to the intrinsic connectivity networks; when integrated, such findings may improve our understanding of the neurobiological basis of PTSD.

\section{Methods}

\section{Participants}

We recruited 86 participants in total; 12 participants withdrew or were excluded, leaving 74 participants who met and completed all study requirements. Exclusion criteria included past or present bipolar disorder, a psychotic disorder or a present substance use disorder. Full remission of previous substance use disorders was required for a minimum of 3 months before study involvement. Any lifetime psychiatric illness or psychotropic medication served as an exclusion criterion for the healthy controls. Finally, nonadherence to $3 \mathrm{~T}$ safety standards, a serious untreated medical illness, pregnancy, a neurobiological or developmental disorder, or a significant head injury involving a loss of consciousness served as additional exclusion criteria.

The final study sample included 56 participants who had a primary diagnosis of PTSD and had been exposed to a potentially morally injurious event, and 18 controls who had been exposed to a potentially morally injurious event but were otherwise healthy. From the participants who had PTSD and had experienced moral injury, we compared 2 subgroups: 28 members of the Canadian Armed Forces (active duty and veterans) and law enforcement officers; and 28 civilians (e.g., paramedics, firefighters, health care workers and victims of interpersonal violence).

All participants provided written, informed consent before study involvement and were compensated financially for their time.

\section{Ethics approval}

Study procedures were approved by the Health Sciences Research Ethics Board at Western University.

\section{Clinical interviews and memory scripts}

We administered clinical questionnaires and state- and traitrelated scales (Appendix 1, available at www.jpn.ca/lookup/ doi/10.1503/jpn.210117/tab-related-content).

We measured moral injury using the Moral Injury Events Scale (MIES), ${ }^{46}$ an 11-item self-report scale developed to assess moral injury-related phenomenology. In the MIES, 9 items address perceptions of violation of moral beliefs or betrayal by self and others, and 2 items address perceptions of trust. Participants are asked to indicate their level of agreement with each statement in relation to their experiences since joining the military on a Likert-type scale ranging from 1 (strongly disagree) to 6 (strongly agree). For the present study, we adapted military-specific language to be more general (e.g., the original statement "I feel betrayed by fellow service members who I once trusted" was adapted to "I feel betrayed by fellow coworkers who I once trusted"). Scores were summed, and a higher score indicated a greater intensity of potentially morally injurious events. To be included in the study, all participants had to have chosen a 6 (strongly agree) for at least 1 statement in the MIES.

We used the MIES to get a general sense of the intensity of the potentially morally injurious events that participants experienced as part of their occupation. Then, we asked participants to think about a specific potentially morally injurious event and a neutral event that took place around the same time. We provided several definitions of moral injury, and then the participant and the research staff had a brief discussion to ensure that the participant had a grasp of the concept and had selected an event that would fit the definition provided (Appendix 1). Importantly, the moral injury-related event - or the specific memory that the participant acknowledged as inducing a moral injury — had to have elicited strong moral emotions when the event took place. In particular, participants were asked to select a moral injury-related event with a moral emotion score of 5 to 8 on a scale of 1 to 10 (10 being most severe). In line with the literature, the presence of strong moral emotions helped to define a moral injury in the present study. We used specific moral injury-related (and neutral) memory events to create 8 short sentences that captured the moments before and during the potentially morally injurious event. These 8 short sentences were read and displayed sentence by sentence in chronological order during fMRI scanning; this served to facilitate memory recall in a step-wise manner in a style consistent with the Autobiographical Interview Administration Manual. ${ }^{47}$ Immediately after each script had been displayed, we collected self-report ratings for shame, guilt and betrayal; we used these as an indication of how strongly each moral emotion was felt during the script-driven memory recall.

Unlike for PTSD, no functional impairment criteria have been developed for moral injury. The presence of moral emotions such as shame, guilt and betrayal - as well as selfrelated processing disturbances such as the inability to forgive oneself and engagement in self-sabotaging behaviours - help to indicate that a person has experienced a moral injury. However, these central features of moral injury do not capture the effect of the moral injury on a person's functioning. To get a sense of impairments related to moral injury, we administered the World Health Organization Disability Assessment Schedule (WHODAS 2.0). ${ }^{48}$ Responses to the WHODAS 2.0 were not factored into the functional impairment criteria for PTSD, so it was not possible to determine whether impairments identified using the WHODAS 2.0 were caused by moral injury or PTSD in participants who had experienced both. 


\section{fMRI image acquisition}

We used a 3 T MRI Scanner (Biograph mMR; Siemens Medical Solutions) for brain imaging, using a Siemens 32-channel head coil locally adapted to the scanner's 4-plug interface. We collected orthogonal scout images and used them to prescribe a 3-dimensional $T_{1}$-weighted anatomical image of the whole head with $1 \mathrm{~mm}$ isotropic resolution (magnetization prepared rapid gradient echo). We acquired functional whole-brain images with blood-oxygen-level-dependent contrast transversely using the manufacturer's gradient echo $\mathrm{T}_{2}{ }^{*}$-weighted blipped-echo-planar sequence (repetition time $3000 \mathrm{~ms}$, echo time $20 \mathrm{~ms}$, field of view $256 \times 256 \mathrm{~mm}$, flip angle $90^{\circ}$, in-plane resolution $2 \times 2 \mathrm{~mm}$, parallel imaging acceleration factor 4 ). Each volume included 60 ascending interleaved slices with a thickness of $2 \mathrm{~mm}$. Participants' heads were stabilized with foam padding, and the experimental runs each consisted of 118 volumes.

\section{fMRI preprocessing}

We preprocessed the functional images in SPM12 (Wellcome Trust Centre for Neuroimaging; www.fil.ion.ucl.ac.uk/spm) using MATLAB R2019 (MathWorks Inc.). Our standard preprocessing routine included discarding 4 initial volumes, reorientation to the axis of the anterior commissure-posterior commissure, spatial alignment to the mean image using rigid body transformation, and reslicing and coregistration of the functional mean image to the participant's anatomical image. Coregistered images were segmented using the "New Segment" method in SPM12. The functional images were then normalized to Montreal Neurological Institute (MNI) space and smoothed with a full width at half maximum Gaussian kernel of $6 \mathrm{~mm}$. We implemented additional correction for motion using the ART software package (Gabrieli Laboratory, McGovern Institute for Brain Research).

\section{Statistical analysis}

\section{Independent component analysis}

Independent component analysis (ICA) refers to a datadriven, computational approach used to determine wholebrain, voxel-wise functional connectivity patterns. ${ }^{49}$ To analyze functional network connectivity across the DMN, we performed a single spatial group ICA using the Group ICA of fMRI Toolbox (GIFT). ${ }^{50}$ We concatenated functional images across all participants $(n=74)$ and task-related conditions into a spatial group ICA to analyze intersubject and intergroup differences in terms of independent component spatial extent and amplitudes. ${ }^{51,52}$ We selected the INFOMAX algorithm and calculated 20 independent components, which we estimated 20 times through ICASSO to ensure reliability. ${ }^{51}$ Then, we back-reconstructed the averaged group independent components using principal component analysis into singleparticipant spatial maps and time courses. ${ }^{53}$

We inspected all 20 independent components visually to confirm that artifacts (e.g., ventricles, white matter, edges) were not present. Then, we correlated all 20 independent components spatially to the DMN mask adopted from the Functional Imaging in Neuropsychiatric Disorders Laboratory database. ${ }^{54}$ The DMN mask included clusters in the medial prefrontal cortex, the anterior cingulate cortex, the posterior cingulate cortex, the precuneus, the thalamus and the superior parietal lobules. We selected the independent component that correlated most to the DMN (DMN IC), and then we combined single-participant spatial maps and time courses across their respective groups to conduct group comparisons in SPM12.

\section{Group differences}

In SPM12, single-participant spatial maps and time courses from the DMN IC were sorted group-wise and added to a 1-way analysis of variance to analyze differences between participants with military- and law enforcement-related PTSD, participants with civilian-related PTSD and healthy controls. We selected group variances as unequal, a more conservative selection that accounted for differences in group size and ensured that statistical power was not biased toward the groups with co-occurring moral injury and PTSD. We generated contrast images and assessed results at a false discovery rate (FDR)-corrected significance threshold of $p_{\mathrm{FDR}}<0.05, k \geq 10$.

We also conducted a region-of-interest analysis to reveal brainstem, midbrain and cerebellar systems more closely. For the region of interest, we used the Spatially Unbiased Infratentorial Template (SUIT) toolbox mask. ${ }^{55}$ The SUIT template mask includes a high-resolution, MNI-normalized cropped cerebellum and brainstem, a large mask and therefore a more conservative selection as a region of interest. We assessed results at a significance threshold of $p_{\mathrm{FDR}}<0.05, k \geq 10$.

\section{Results}

\section{Demographics and clinical measures}

Group demographics and clinical characteristics are provided in Table 1.

We found significant differences between groups for age, but closer examination of the influence of age on the bloodoxygen-level-dependent signal revealed that the difference was nonsignificant. The PTSD groups reported higher scores on the Childhood Trauma Questionnaire, although the entire sample had been exposed to childhood maltreatment or neglect (as measured by any subscale $>5$, or minimization scale $>0$ ). As expected, the PTSD groups reported significantly higher scores on the Multiscale Dissociation Inventory, MIES and WHODAS 2.0 as compared to healthy controls.

\section{Component selection}

In GIFT, independent components $3(r=0.379)$ and $9(r=$ $0.371)$ were correlated more strongly with the DMN mask than any of the other independent components. Independent component 3 included anterior (e.g., medial prefrontal cortex, anterior cingulate cortex) and posterior (e.g., posterior cingulate cortex, precuneus) DMN regions, but independent component 9 included anterior DMN regions only. 


\begin{tabular}{|c|c|c|c|}
\hline Characteristic $^{*}$ & $\begin{array}{l}\text { Military- and law- } \\
\text { enforcement-related } \\
\text { PTSD }\end{array}$ & Civilian-related PTSD & $\begin{array}{l}\text { Healthy controls exposed } \\
\text { to a moral injury event }\end{array}$ \\
\hline Participants, $n$ & 28 & 28 & 18 \\
\hline $\mathrm{M} / \mathrm{F}, n$ & $25 / 3$ & $9 / 19$ & $7 / 11$ \\
\hline Age, yr & $49 \pm 8$ & $49 \pm 8$ & $33 \pm 11$ \\
\hline Clinician-Administered PTSD Scale, total score & $40.9 \pm 7.9 \dagger$ & $41.7 \pm 6.6 \dagger$ & $0 \pm 0$ \\
\hline Clinician-Administered PTSD Scale, criterion D & $15.4 \pm 3.5 \dagger$ & $15.4 \pm 3.2 \dagger$ & $0 \pm 0$ \\
\hline Childhood Trauma Questionnaire, total score & $52.8 \pm 24.2 \dagger$ & $65.7 \pm 23.4 \dagger$ & $30.3 \pm 8.4$ \\
\hline Multiscale Dissociation Inventory, total score & $60.3 \pm 16.3 \dagger$ & $68.0 \pm 17.8 \dagger$ & $35.6 \pm 5.2$ \\
\hline Moral Injury Events Scale, total score & $39.9 \pm 6.9 \dagger$ & $39.3 \pm 7.7 \dagger$ & $25.9 \pm 9.6$ \\
\hline World Health Organization Disability Assessment Schedule 2.0, total score & $30.3 \pm 9.3 \dagger$ & $31.6 \pm 8.7 \dagger$ & $12.9 \pm 1.7$ \\
\hline Major depressive disorder, recurrent, $n$ & $\begin{array}{l}\text { Current: } 9 \\
\text { Past: } 0\end{array}$ & $\begin{array}{l}\text { Current: } 21 \\
\text { Past: } 0\end{array}$ & $\begin{array}{l}\text { Current: } 0 \\
\text { Past: } 0\end{array}$ \\
\hline Major depressive disorder, single episode, $n$ & $\begin{array}{l}\text { Current: } 4 \\
\text { Past: } 1\end{array}$ & $\begin{array}{l}\text { Current: } 0 \\
\text { Past: } 0\end{array}$ & $\begin{array}{l}\text { Current: } 0 \\
\text { Past: } 0\end{array}$ \\
\hline Prescribed psychotropic medication, $n \ddagger$ & 23 & 10 & 0 \\
\hline \multicolumn{4}{|c|}{$\begin{array}{l}\mathrm{F}=\text { female; } \mathrm{M}=\text { male; } \mathrm{PTSD}=\text { posttraumatic stress disorder. } \\
\text { *Values are mean } \pm \text { standard deviation, unless otherwise indicated. } \\
\text { †Significantly higher clinical symptom values relative to healthy controls, based on } 1 \text {-way analysis of variance and follow-up } t \text { tests }(p<0.05) . \\
\ddagger \text { See Appendix } 1 \text { for a full breakdown of the psychotropic medications prescribed, the class they belonged to, and how many past and present prescriptions were recorded in each group. }\end{array}$} \\
\hline
\end{tabular}

Next, independent components 3 and 9 were correlated temporally with onsets of the neutral and the moral injury event-related tasks. Independent components $3(r=0.177)$ and $9(r=0.167)$ showed similar correlations, but we ultimately selected independent component 3 as the DMN IC (Figure 1) because it revealed the strongest correlations and the greatest overlap with the DMN mask.

\section{Group differences}

Participants with civilian-related PTSD revealed stronger precuneus $\left(x, y, z=14,-46,54 ; t_{71}=5.67 ; p_{\mathrm{FDR}}=0.009 ; k=43\right)$ functional network connectivity across the DMN as compared to participants with military- and law enforcementrelated PTSD (Table 2). In the region-of-interest output, we observed stronger PAG $\left(x, y, z=0,-32,-8 ; t_{71}=4.95\right.$; $\left.p_{\mathrm{FDR}}=0.028 ; k=39\right)$ and cerebellar lobule IX $(x, y, z=10,-66$, $\left.-40 ; t_{71}=4.44 ; p_{\mathrm{FDR}}=0.046 ; k=49\right)$ functional network connectivity in participants with civilian-related PTSD as compared to healthy controls. Finally, we observed a trend toward stronger PAG $\left(x, y, z=4,-30,-2 ; t_{71}=4.22 ; p_{\mathrm{FDR}}=0.076\right.$; $k=60$ ) functional network connectivity in participants with military- and law enforcement-related PTSD as compared to healthy controls.

\section{Clinical correlations}

In participants with military- and law enforcement-related PTSD, we observed a significant negative correlation between precentral gyrus functional network connectivity and state shame scores $\left(x, y, z=-36,-28,40 ; t_{27}=8.29 ; p_{\mathrm{FDR}}=\right.$ $0.049 ; k=24)$. In the region-of-interest output, we observed a significant positive correlation between cerebellar lobule IX functional network connectivity and state shame scores in participants with military- and law enforcement-related $\operatorname{PTSD}\left(x, y, z=2,-56,-24 ; t_{27}=8.10 ; p_{\mathrm{FDR}}=0.006 ; k=24\right)$. Finally, we observed a significant positive correlation between cerebellar lobule VII functional network connectivity $\left(x, y, z=42,-60,-34 ; t_{55}=5.10 ; p_{\mathrm{FDR}}=0.041 ; k=28\right)$ and total scores on the Clinician-Administered PTSD Scale in participants with PTSD $(n=56)$, and a significant negative correlation between brainstem functional network connectivity $(x, y$, $\left.z=14,-16,-18 ; t_{55}=4.96 ; p_{\mathrm{FDR}}=0.049 ; k=19\right)$ and ClinicianAdministered PTSD Scale total scores (Table 3).

\section{Discussion}

We sought to identify the neural correlates that underlie moral injury in people with and without PTSD, with a particular focus on brainstem, midbrain and cerebellar systems. We revealed stronger midbrain and cerebellar functional network connectivity across the DMN in participants with civilianrelated PTSD as compared to healthy controls who had been exposed to a potentially morally injurious event, as well as a trend toward stronger midbrain functional network connectivity in participants with military- and law enforcementrelated PTSD as compared to healthy controls. Similar to functional connectivity, functional network connectivity examines temporal correlations among distinct brain regions, only at the network level. An ICA helped to define the independent component that was most correlated spatially with the DMN, taking into consideration the experimental time series (Figure 2). We conducted the ICA across the entire sample to make group comparisons within the same network; otherwise, we would not have been able to assess group-wise statistical differences in the DMN. For the study task, we developed scripts based on an emotionally evocative potentially morally injurious event (and a neutral event); 


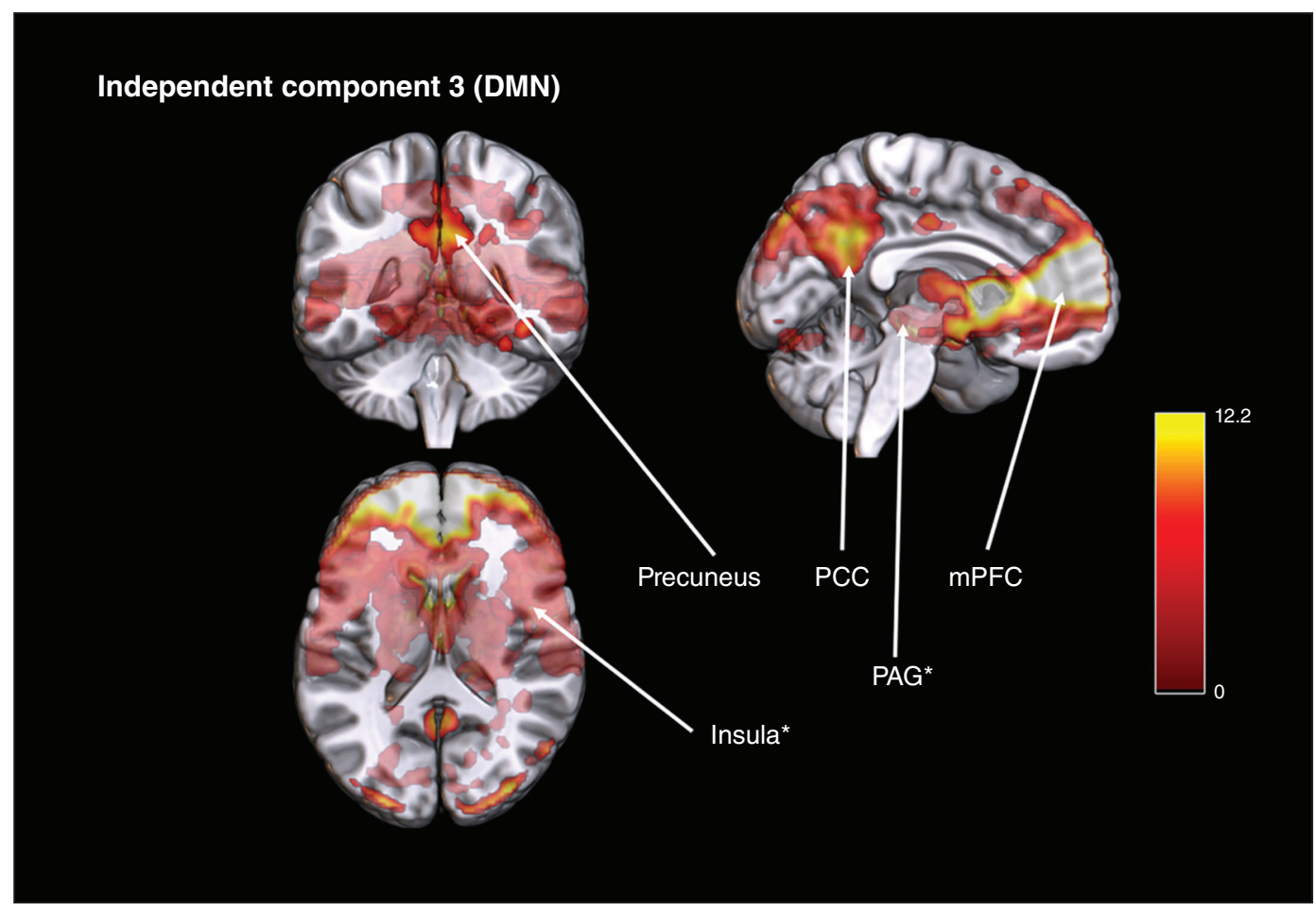

Figure 1: In GIFT, independent component 3 (shown above) was correlated very strongly with the DMN mask. Independent component 3 included key DMN-related hubs, such as the MPFC, the PCC and the precuneus. It also included supplementary DMN-related areas, such as the middle cingulate cortex and the bilateral temporal lobes, but these areas revealed less functional network connectivity than the hubs. We also observed clusters in the insula, midbrain and cerebellum, which are not generally thought to be involved in the DMN (indicated by an asterisk). DMN = default mode network; fMRI = functional MRI; GIFT = Group ICA of fMRI Toolbox; ICA = independent component analysis; $\mathrm{mPFC}=$ medial prefrontal cortex; $\mathrm{PAG}=$ periaqueductal grey; $\mathrm{PCC}=$ posterior cingulate cortex .

Table 2: Group differences: functional network connectivity*

\begin{tabular}{|c|c|c|c|c|c|c|c|}
\hline $\begin{array}{l}\text { Independent } \\
\text { component }\end{array}$ & Contrast & Brain region & Cluster, $k$ & $\begin{array}{c}\text { MNI coordinates, } \\
x, y, z\end{array}$ & $t$ statistic & Z score & $\begin{array}{c}p_{\mathrm{FDR}} \\
(\mathrm{peak})\end{array}$ \\
\hline $\begin{array}{l}\text { Independent } \\
\text { component 3: DMN } \\
\text { (whole brain) }\end{array}$ & $\begin{array}{c}\text { Civilian-related PTSD > } \\
\text { military- and law-enforcement- } \\
\text { related PTSD }\end{array}$ & Precuneus & 43 & $14,-46,54$ & 5.67 & 5.38 & 0.009 \\
\hline \multirow{3}{*}{$\begin{array}{l}\text { Independent } \\
\text { component 3: DMN } \\
\text { (region of interest) }\end{array}$} & \multirow{2}{*}{$\begin{array}{c}\text { Civilian-related PTSD > } \\
\text { healthy controls }\end{array}$} & Periaqueductal grey & 39 & $0,-32,-8$ & 4.95 & 4.75 & 0.028 \\
\hline & & Cerebellar lobule VII & 49 & $10,-66,-40$ & 4.44 & 4.29 & 0.046 \\
\hline & $\begin{array}{l}\text { Military- and law-enforcement- } \\
\text { related PTSD > healthy controls }\end{array}$ & Periaqueductal grey & 60 & $4,-30,-2$ & 4.22 & 4.09 & $0.076 \dagger$ \\
\hline
\end{tabular}

DMN = default mode network; FDR = false discovery rate; MNI = Montreal Neurological Institute; PTSD = posttraumatic stress disorder.

${ }^{*}$ Group-level functional network connectivity across the independent component correlated to the DMN is displayed for whole-brain and region-of-interest analyses; $t$ contrasts were evaluated at a significance threshold of $p_{\mathrm{FDR}}<0.05, k>10$ (peak-corrected).

†Denotes a group difference approaching significance.

using this task, we hoped to reveal the neural correlates that underpin moral injury during memory recall and self-reflection. Taken together, these novel findings provide strong evidence that in PTSD, the DMN (a network dominated by higherlevel, cortical systems) may be biased toward lower-level, midbrain systems. Exactly how midbrain systems alter DMN-mediated processing remains to be explored, but we suspect that these effects underpin, at least in part, how moral injuries are represented in relation to the sense of self.

\section{Midbrain functional network connectivity}

The DMN supports autobiographical memory and self-related processing. ${ }^{44,45}$ During script-driven, moral injury-related memory recall, we found that the DMN had greater midbrain functional network connectivity among participants with both moral injury and PTSD as compared to controls who had been exposed to a potentially morally injurious event but were otherwise healthy. Although research assessing brainstem, 
Table 3: Clinical correlations: functional network connectivity

\begin{tabular}{|c|c|c|c|c|c|c|c|c|}
\hline $\begin{array}{l}\text { Independent } \\
\text { component }\end{array}$ & Group & Clinical score (direction) & Brain region & $\begin{array}{c}\text { Cluster, } \\
k\end{array}$ & $\begin{array}{c}\text { MNI coordinates, } \\
x, y, z\end{array}$ & $t$ statistic & $Z$ score & $\begin{array}{c}p_{\mathrm{FDR}} \\
\text { (peak) }\end{array}$ \\
\hline $\begin{array}{l}\text { Independent } \\
\text { component 3: DMN } \\
\text { (whole brain) }\end{array}$ & $\begin{array}{l}\text { Military- and law- } \\
\text { enforcement- } \\
\text { related PTSD }\end{array}$ & State: shame (-) & $\begin{array}{l}\text { Precentral } \\
\text { gyrus }\end{array}$ & 24 & $-36,-28,40$ & 8.29 & 5.18 & 0.049 \\
\hline \multirow[t]{3}{*}{$\begin{array}{l}\text { Independent } \\
\text { component 3: DMN } \\
\text { (region of interest) }\end{array}$} & $\begin{array}{l}\text { Military- and law- } \\
\text { enforcement- } \\
\text { related PTSD }\end{array}$ & State: shame (+) & $\begin{array}{l}\text { Cerebellar } \\
\text { lobule IX }\end{array}$ & 24 & $2,-56,-24$ & 8.10 & 5.12 & 0.006 \\
\hline & PTSD & $\begin{array}{l}\text { Clinician-Administered } \\
\text { PTSD Scale, total score }(+)\end{array}$ & $\begin{array}{l}\text { Cerebellar } \\
\text { lobule VII }\end{array}$ & 28 & $42,-60,-34$ & 5.10 & 4.58 & 0.041 \\
\hline & & $\begin{array}{c}\text { Clinician-Administered } \\
\text { PTSD Scale, total score (-) }\end{array}$ & Brainstem & 19 & $14,-16,-18$ & 4.96 & 4.48 & 0.049 \\
\hline
\end{tabular}

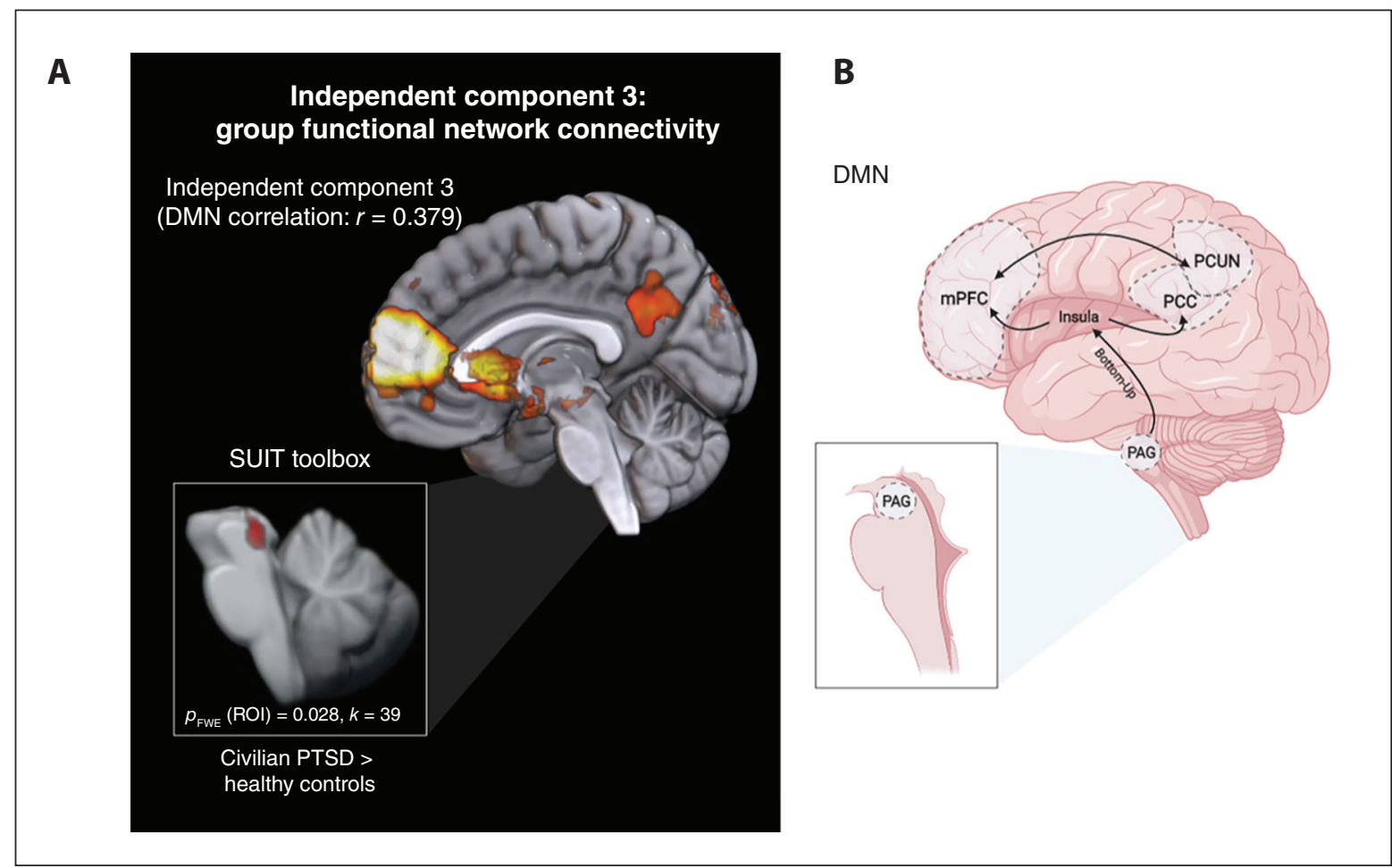

Figure 2: (A) Top: the whole-brain image shows a thresholded independent component 3 ( $k>35$ cut-off) and the DMN-related spatial correlation value. Bottom: midbrain PAG functional network connectivity clusters that were more strongly involved in the DMN in participants with civilian-related PTSD versus healthy controls exposed to moral injury have been superimposed onto the cropped brainstem and cerebellum mask from the SUIT toolbox. (B) This summary image interprets the functional connectivity revealed in the present study and the study by Terpou and colleagues. ${ }^{43}$ In PTSD, we suspect that the DMN-related hubs - namely the mPFC, PCC and precuneus - reveal strong functional connectivity during DMN-mediated processing, similar to healthy controls. However, Terpou and colleagues ${ }^{43}$ found stronger PAG-directed functional connectivity to DMN-related hubs in PTSD, a finding that converges with the stronger PAG functional network connectivity we found in the present study. Taking these findings together, we suggest that the PAG may be driving the DMN in people with PTSD, primarily in a bottom-up manner; functional connectivity is mediated by the insula and directed toward the DMN. DMN = default mode network; FWE = family-wise error; $\mathrm{mPFC}=$ medial prefrontal cortex; $\mathrm{PAG}=$ periaqueductal grey; $\mathrm{PCC}=$ posterior cingulate cortex; $\mathrm{PCUN}=$ precuneus; PTSD = posttraumatic stress disorder; ROI = region of interest; SUIT = Spatially Unbiased Infratentorial Template.

midbrain and cerebellar involvement in the intrinsic connectivity networks is limited, midbrain and DMN-related functional connectivity has been reported elsewhere in PTSD. In particular, Terpou and colleagues ${ }^{43}$ observed that, as compared to healthy controls, participants with PTSD revealed stronger midbrain PAG-directed functional connectivity to anterior and posterior DMN-related hubs during subliminal, trauma-related stimulus processing. This study 
used a different task and a different sample, but their task and the one employed in the present study (which relied largely on the DMN) both engaged the midbrain PAG more strongly in participants with PTSD. In people with PTSD, these results may suggest that DMN-mediated memory recall and selfrelated processing is biased toward innately conserved midbrain systems. Recruited more commonly during threat- and trauma-related processing, midbrain systems may be the reason why participants with both moral injury and PTSD reported higher levels of shame, guilt and betrayal during moral injury event-related memory recall. These moral emotions depend on viscerosensory pathways coordinated by the midbrain, $33,34,56$ which, through coactivation with the DMN, may have produced the functional network connectivity patterns we observed.

These findings raise the question of whether such midbrainDMN connectivity patterns persist at rest. If they do, these results may suggest a neurobiological basis for the persistent shame experienced by people with PTSD, a phenomenon that perpetuates moral injury. ${ }^{17-19}$ More broadly, these results speak to self-related processing disturbances. In PTSD, self- and trauma-related processing become intimately linked. For example, Sutherland and Bryant ${ }^{57}$ revealed that participants with PTSD, when asked to describe a self-defining memory, are more likely to describe a trauma-related memory, highlighting the centrality trauma has for self-identity in PTSD. Such self-related processing disturbances naturally implicate the DMN. However, given that we observed DMN-related differences more in the midbrain, it may be that midbrain systems also contribute to self-related processing disturbances. Because higher-level, self-related concepts (e.g., self-identity, body image) rely on lower-level, bodily self-awareness (e.g., interoceptive sensibility) as a scaffold, ${ }^{32,58}$ these lower-level processes and the systems that underlie them could partially explain self-related disturbances at a more cognitive level, a hypothesis that warrants further study.

\section{Cerebellar functional network connectivity}

Unexpectedly, we revealed stronger cerebellar lobule IX functional network connectivity across the DMN in participants with civilian-related PTSD as compared to healthy controls. As well, in participants with military- and lawenforcement-related PTSD, we revealed a positive correlation between cerebellar lobule IX functional network connectivity and moral injury-related, state shame scores. Although the DMN has been thought to be represented largely cortically, Habas and colleagues ${ }^{59}$ found that the DMN had distinct correlates in the cerebellum. Specifically, they conducted a resting-state ICA in a healthy sample and found that the cerebellar lobule IX was connected to the DMN. In their discussion of these findings, the authors referenced previous work by Addis and colleagues ${ }^{60}$ that revealed cerebellar lobule IX activation during past- and future-related event memory recall, in parallel with activation in the precuneus and the retrosplenial cortex. Taken together, the findings of the present study and those of earlier studies suggest that cerebellar lobule IX functional connectivity to the DMN may extend beyond the resting state.

Notably, altered cerebellar lobule IX functional connectivity across the DMN has also been revealed in schizophrenia and somatic symptom disorder. Guo and colleagues ${ }^{61}$ and Wang and colleagues ${ }^{62}$ reported stronger cerebellar lobule IX functional connectivity with the DMN in drug-naive participants with first-episode schizophrenia and in participants with somatic symptom disorder as compared to healthy controls. We know that PTSD, schizophrenia and somatic symptom disorder all feature exaggerated bottom-up processing and related perceptual symptoms, such as hypervigilance in PTSD, hallucinations in schizophrenia and preoccupation with physical pain and fatigue in somatic symptom disorder. ${ }^{63}$ Although these symptoms present differently, they are all mediated by overactive bottom-up autonomic and sensory systems, as well as concomitant underactive top-down modulatory systems. ${ }^{63,64}$

\section{Future directions}

Based on contrasting results between participants with militaryand law enforcement-related PTSD and participants with civilian-related PTSD, we encourage future researchers to study occupational differences in moral codes and examine how these differences relate to moral injury. As well, we encourage future researchers to study more racially diverse samples to explore whether occupational moral injury differs based on race; it may be that moral injury is more common among socially, economically and educationally disadvantaged groups. Finally, despite early work by Barnes and colleagues, ${ }^{23}$ Lloyd and colleagues ${ }^{25}$ and others, ${ }^{1-22}$ more research is needed to disentangle the neural correlates that underlie moral injury and PTSD. We encourage future research to study how interrelated these constructs are. Any study assessing moral injury and PTSD runs into a similar problem with control groups. In PTSD, trauma can be defined clearly, leading to a straightforward definition of what constitutes trauma-exposed but otherwise healthy controls. Because moral injury has been defined as involving a biopsychosocial injury, describing moral injury-exposed but otherwise healthy controls is more difficult, especially because such a definition requires a concrete understanding of what exactly is meant by "injury."

\section{Limitations}

The present study had several limitations. First, although fMRI affords high spatial resolution, resolution procedures are largely based on grey and white matter distributions at the cortical level, which have a more easily discernible delineation. Second, we did not identify participants with PTSD who met dissociative criteria. Dissociative symptomatology has distinct functional connectivity patterns compared to PTSD, ${ }^{64}$ which may have added group-level heterogeneity to the PTSD groups. Third, many participants with civilian-related PTSD and a few participants with militaryand law enforcement-related PTSD had recurrent major 
depressive disorder. The DMN has been implicated in the pathology of major depressive disorder, where the network generally displays greater within-network functional connectivity. ${ }^{65,66}$ We decided to include participants with PTSD who had recurrent major depressive disorder, sacrificing control to gain generalizability. Fourth, many participants with co-occurring moral injury and PTSD had been prescribed psychotropic medication. These medications act variously on different receptors and neurotransmitters and may have served as a confound. Finally, moral injury is an evolving construct, and, although we administered standard psychometric scales to assess for this condition, a new standard may emerge in time.

\section{Conclusion}

Collectively, these results strongly suggest that midbrain systems are not only involved in the DMN, but are the systems in which differences between participants with PTSD and healthy controls are most pronounced. Given the role of the DMN in self-related processing, these results may assist in explaining the self-related processing disturbances reported commonly in PTSD, including the viscerosensory urge to cringe, hide or withdraw and to engage in ruminative, self-critical thoughts when recalling potentially morally injurious events. More generally, these results highlight the need to expand on current corticocentric models that predominate in mental health research to include brainstem, midbrain and cerebellar systems, which are altered in people with PTSD but are comparatively underinvestigated. Finally, these results suggest that bottom-up psychotherapy approaches should be considered strongly as an adjunct to top-down psychotherapy. Bottom-up approaches generally focus on lower-order brainstem and midbrain systems, and serve to direct awareness to the body and the precognitive (and in some cases, preaffective) physical phenomena that precede higher-level processing. Sensorimotor therapy, deep brain reorienting and neurofeedback have been implemented successfully in PTSD and (when conducted alongside topdown psychotherapy) provide a more integrated, embodied cognitive approach to therapy.

Acknowledgements: The authors would like to thank all the individuals who participated, as well as Homewood Health in Guelph, Ontario, Canada, who facilitated referrals. We are also grateful to our dedicated research and clinical team, without whom we could not have done this work.

Affiliations: From the Department of Neuroscience (Terpou, Neufeld), the Department of Psychiatry (Lloyd, Densmore, Théberge, Neufeld, Lanius), the Department of Medical Biophysics (Théberge), the Department of Psychology (Neufeld), Western University, London, Ont.; the Imaging Division, Lawson Health Research Institute (Densmore, Lanius), the Department of Psychology, Neuroscience, and Behaviour (Lloyd), the Department of Psychiatry and Behavioural Neurosciences (McKinnon), McMaster University, Hamilton, Ont.; Mood Disorders Program, St. Joseph's Healthcare (McKinnon), Hamilton, Ont.; Homewood Research Institute (McKinnon, Lanius), Guelph, Ont.; Canadian Forces, Health Services (Jetly), Ottawa, Ont.
Funding: This work was supported by infrastructure funds from the Canada Foundation for Innovation Grant (Théberge; grant number: 31724) and Lawson Health Research Institute, as well as operating funds from the Canadian Institute of Military and Veteran Health Research, Green Shield Canada, the Centre of Excellence on PTSD, Canada, and the Canadian Institutes of Health Research (McKinnon and Lanius; grant number: 148784). R. Lanius is supported by the Harris-Woodman Chair in Psyche and Soma at Western University, and M. McKinnon is supported by the Homewood Chair in Mental Health and Trauma at McMaster University. B. Terpou is supported by the Jonathan and Joshua Memorial Scholarship and Mitacs Elevate funding, partnered generously by Homewood Research Institute.

Competing interests: No other competing interests were declared.

Contributors: R. Jetly and R. Lanius designed the study. C. Lloyd and J. Théberge acquired the data, which B. Terpou, M. Densmore, M. McKinnon and R. Neufeld analyzed. B. Terpou, C. Lloyd and R. Lanius wrote the article, which M. Densmore, M. McKinnon, J. Théberge, R. Neufeld and R. Jetly reviewed. All authors approved the final version to be published and can certify that no other individuals not listed as authors have made substantial contributions to the paper.

Content licence: This is an Open Access article distributed in accordance with the terms of the Creative Commons Attribution (CC BY-NC-ND 4.0) licence, which permits use, distribution and reproduction in any medium, provided that the original publication is properly cited, the use is noncommercial (i.e., research or educational use), and no modifications or adaptations are made. See: https://creativecommons.org/licenses/by-nc-nd/4.0/

\section{References}

1. Litz BT, Stein N, Delaney E, et al. Moral injury and moral repair in war veterans: a preliminary model and intervention strategy. Clin Psychol Rev 2009;29:695-706.

2. Held P, Klassen BJ, Brennan MB, et al. Using prolonged exposure and cognitive processing therapy to treat veterans with moral injurybased PTSD: two case examples. Cogn Behav Pract 2018;25:377-90.

3. Jordan AH, Eisen E, Bolton E, et al. Distinguishing war-related PTSD resulting from perpetration- and betrayal-based morally injurious events. Psychol Trauma 2017;9:627-34.

4. Lancaster SL. Negative outcomes after morally injurious experiences: a replication and extension. Psychol Trauma 2018;10:456-62.

5. Borges LM, Holliday R, Barnes SM, et al. A longitudinal analysis of the role of potentially morally injurious events on COVID19-related psychosocial functioning among healthcare providers. PLoS One 2021;16:e260033.

6. Griffin BJ, Purcell N, Burkman K, et al. Moral injury: an integrative review. J Trauma Stress 2019;32:350-62.

7. Farnsworth JK, Drescher KD, Nieuwsma JA, et al. The role of moral emotions in military trauma: implications for the study and treatment of moral injury. Rev Gen Psychol 2014;18:249-62.

8. Browne KC, Trim RS, Myers US, et al. Trauma-related guilt: conceptual development and relationship with posttraumatic stress and depressive symptoms. J Trauma Stress 2015;28:134-41.

9. Bryan CJ, Bryan ABO, Roberge E, et al. Moral injury, posttraumatic stress disorder, and suicidal behavior among national guard personnel. Psychol Trauma 2018;10:36-45.

10. Currier JM, McDermott RC, Farnsworth JK, et al. Temporal associations between moral injury and posttraumatic stress disorder symptom clusters in military veterans. J Trauma Stress 2019;32:382-92.

11. Protopopescu A, Boyd JE, O'Connor C, et al. Examining the associations among moral injury, difficulties with emotion regulation, and symptoms of PTSD, depression, anxiety, and stress among Canadian military members and veterans: a preliminary study. J Mil Veteran Fam Health 2021;7:72-80. 
12. Purcell N, Koenig CJ, Bosch J, et al. Veterans' perspectives on the psychosocial impact of killing in war. Couns Psychol 2016;44: 1062-99.

13. Litz BT, Contractor AA, Rhodes C, et al. Distinct trauma types in military service members seeking treatment for posttraumatic stress disorder. J Trauma Stress 2018;31:286-95.

14. Maguen S, Metzler TJ, Bosch J, et al. Killing in combat may be independently associated with suicidal ideation. Depress Anxiety 2012;29:918-23.

15. Greenberg N, Docherty M, Gnanapragasam S, et al. Managing mental health challenges faced by healthcare workers during COVID-19 pandemic. BMJ 2020;368:m1211.

16. Bryan $\mathrm{CJ}$, Bryan $\mathrm{ABO}$, Anestis $\mathrm{MD}$, et al. Measuring moral injury: psychometric properties of the Moral Injury Events Scale in two military samples. Assessment 2016;23:557-70.

17. Cloitre M, Cohen LR, Koenen KC. Treating survivors of childhood abuse: pyschotherapy for interrupted life. New York: Guilford Press, 2006.

18. Nazarov A, Jetly R, McNeely H, et al. Role of morality in the experience of guilt and shame within the armed forces. Acta Psychiatr Scand 2015;132:4-19.

19. Crocker LD, Haller M, Norman SB, et al. Shame versus trauma-related guilt as mediators of the relationship between PTSD symptoms and aggression among returning veterans. Psychol Trauma 2016;8:520-7

20. Norman SB, Wilkins KC, Myers US, et al. Trauma informed guilt reduction therapy with combat veterans. Cogn Behav Pract 2014;21: 78-88.

21. Norman SB, Haller M, Kim HM, et al. Trauma related guilt cognitions partially mediate the relationship between PTSD symptom severity and functioning among returning combat veterans. J Psychiatr Res 2018;100:56-62.

22. Roth SL, Andrews K, Protopopescu A, et al. Mental health symptoms in public safety personnel: examining the effects of adverse childhood experiences and moral injury. Child Abuse Negl 2022;123: 105394.

23. Barnes HA, Hurley RA, Taber KH. Moral injury and PTSD: often co-occurring yet mechanistically different. J Neuropsychiatry Clin Neurosci 2019;31:A4-103.

24. Sun D, Phillips RD, Mulready HL, et al. Resting-state brain fluctuation and functional connectivity dissociate moral injury from posttraumatic stress disorder. Depress Anxiety 2019;36:442-52.

25. Lloyd CS, Nicholson AA, Densmore M, et al. Shame on the brain: neural correlates of moral injury event recall in posttraumatic stress disorder. Depress Anxiety 2021;38:596-605.

26. Rabellino D, Tursich M, Frewen PA, et al. Intrinsic connectivity networks in post-traumatic stress disorder during sub- and supraliminal processing of threat-related stimuli. Acta Psychiatr Scand 2015;132:365-78

27. Tursich M, Ros T, Frewen PA, et al. Distinct intrinsic network connectivity patterns of post-traumatic stress disorder symptom clusters. Acta Psychiatr Scand 2015;132:29-38

28. Lanius RA, Frewen PA, Tursich M, et al. Restoring large-scale brain networks in PTSD and related disorders: a proposal for neuroscientifically informed treatment interventions. Eur J Psychotraumatol 2015;6:27313.

29. Terpou BA, Densmore M, Theberge J, et al. The threatful self: midbrain functional connectivity to cortical midline and parietal regions during subliminal trauma-related processing in PTSD. Chronic Stress (Thousand Oaks) 2019;3:2470547019871369.

30. Terpou BA, Densmore M, Thome J, et al. The innate alarm system and subliminal threat presentation in posttraumatic stress disorder: neuroimaging of the midbrain and cerebellum. Chronic Stress (Thousand Oaks) 2019;3:2470547018821496.

31. Dorahy MJ. The impact of dissociation, shame, and guilt on interpersonal relationships in chronically traumatized individuals: a pilot study. J Trauma Stress 2010;23:653-6.
32. Lanius R, Frewen P, Nazarov A, et al. A social cognitive neuroscience approach to PTSD: clinical and research perspectives. In: Lanius UF, Paulsen SL and Corrigan FM, editors. Neurobiology and treatment of traumatic dissociation: towards an embodied self. New York: Springer; 2014: 69-80.

33. Panksepp J. The neuro-evolutionary cusp between emotions and cognitions. Consciousness Emotion 2000;1:15-54.

34. Panksepp J. The basic emotional circuits of mammalian brains: do animals have affective lives? Neurosci Biobehav Rev 2011;35:1791-804.

35. De Oca BM, DeCola JP, Maren S, et al. Distinct regions of the periaqueductal gray are involved in the acquisition and expression of defensive responses. J Neurosci 1998;18:3426-32.

36. Coimbra NC, De Oliveira R, Freitas RL, et al. Neuroanatomical approaches of the tectum-reticular pathways and immunohistochemical evidence for serotonin-positive perikarya on neuronal substrates of the superior colliculus and periaqueductal gray matter involved in the elaboration of the defensive behavior and fear-induced analgesia. Exp Neurol 2006;197:93-112.

37. Brandão ML, Zanoveli JM, Ruiz-Martinez RC, et al. Different patterns of freezing behavior organized in the periaqueductal gray of rats: association with different types of anxiety. Behav Brain Res 2008;188:1-13

38. Harricharan S, Rabellino D, Frewen PA, et al. fMRI functional connectivity of the periaqueductal gray in PTSD and its dissociative subtype. Brain Behav 2016;6:e00579.

39. Nicholson AA, Friston KJ, Zeidman P, et al. Dynamic causal modeling in PTSD and its dissociative subtype: bottom-up versus topdown processing within fear and emotion regulation circuitry. Hum Brain Mapp 2017;38:5551-61.

40. Webb EK, Huggins AA, Belleau EL, et al. Acute posttrauma restingstate functional connectivity of periaqueductal gray prospectively predicts posttraumatic stress disorder symptoms. Biol Psychiatry Cogn Neurosci Neuroimaging 2020;5:891-900.

41. Felmingham K, Williams LM, Kemp AH, et al. Neural responses to masked fear faces: sex differences and trauma exposure in posttraumatic stress disorder. J Abnorm Psychol 2010;119:241-7.

42. Steuwe C, Daniels JK, Frewen PA, et al. Effect of direct eye contact in PTSD related to interpersonal trauma: an fMRI study of activation of an innate alarm system. Soc Cogn Affect Neurosci 2014;9:88-97.

43. Terpou BA, Densmore M, Theberge J, et al. The hijacked self: disrupted functional connectivity between the periaqueductal gray and the default mode network in posttraumatic stress disorder using dynamic causal modeling. Neuroimage Clin 2020;27:102345.

44. Qin P, Northoff G. How is our self related to midline regions and the default-mode network? Neuroimage 2011;57:1221-33.

45. Raichle ME. The brain's default mode network. Annu Rev Neurosci 2015;38:433-47.

46. Nash WP, Marino Carper TL, Mills MA, et al. Psychometric evaluation of the moral injury events scale. Mil Med 2013;178:646-52.

47. Levine B, Svoboda E, Hay JF, et al. Aging and autobiographical memory: dissociating episodic and semantic retrieval. Psychol Aging 2002;17:677-89.

48. Üstün TB, Kostanjsek N, Chatterii S, et al, editors. Measuring health and disability: manual for WHO Disability Assessment Schedule (WHODAS 2.0). Geneva: World Health Organization; 2010.

49. Calhoun VD, Liu J, Adali T. A review of group ICA for fMRI data and ICA for joint inference of imaging, genetic, and ERP data. Neuroimage 2009;45:S163-72.

50. Calhoun VD, Adali T, Pearlson GD, et al. A method for making group inferences from functional MRI data using independent component analysis. Hum Brain Mapp 2001;14:140-51.

51. Allen EA, Erhardt EB, Wei Y, et al. Capturing inter-subject variability with group independent component analysis of fMRI data: a simulation study. Neuroimage 2012;59:4141-59.

52. Ros T, Theberge J, Frewen PA, et al. Mind over chatter: plastic upregulation of the fMRI salience network directly after EEG neurofeedback. Neuroimage 2013;65:324-35. 
53. Calhoun VD, Adali T. Multisubject independent component analysis of fMRI: a decade of intrinsic networks, default mode, and neurodiagnostic discovery. IEEE Rev Biomed Eng 2012;5:60-73.

54. Shirer WR, Ryali S, Rykhlevskaia E, et al. Decoding subjectdriven cognitive states with whole-brain connectivity patterns. Cereb Cortex 2012;22:158-65.

55. Diedrichsen J. A spatially unbiased atlas template of the human cerebellum. Neuroimage 2006;33:127-38.

56. Harenski CL, Hamann S. Neural correlates of regulating negative emotions related to moral violations. Neuroimage 2006;30:313-24.

57. Sutherland K, Bryant RA. Self-defining memories in post-traumatic stress disorder. Br J Clin Psychol 2005;44:591-8.

58. Quigley KS, Kanoski S, Grill WM, et al. Functions of interoception: from energy regulation to experience of the self. Trends Neurosci 2021;44:29-38

59. Habas C, Kamdar N, Nguyen D, et al. Distinct cerebellar contributions to intrinsic connectivity networks. J Neurosci 2009;29:8586-94.

60. Addis DR, Wong AT, Schacter DL. Remembering the past and imagining the future: common and distinct neural substrates during event construction and elaboration. Neuropsychologia 2007;45:1363-77.
61. Guo W, Liu F, Zhang Z, et al. Increased cerebellar functional connectivity with the default-mode network in unaffected siblings of schizophrenia patients at rest. Schizophr Bull 2015;41:1317-25.

62. Wang H, Guo W, Liu F, et al. Clinical significance of increased cerebellar default-mode network connectivity in resting-state patients with drug-naive somatization disorder. Medicine (Baltimore) 2016;95:e4043.

63. Wigman JT, van Os J, Borsboom D, et al. Exploring the underlying structure of mental disorders: cross-diagnostic differences and similarities from a network perspective using both a top-down and a bottom-up approach. Psychol Med 2015;45:2375-87.

64. Lanius RA, Vermetten E, Loewenstein RJ, et al. Emotion modulation in PTSD: clinical and neurobiological evidence for a dissociative subtype. Am J Psychiatry 2010;167:640-7.

65. Hamilton JP, Etkin A, Furman DJ, et al. Functional neuroimaging of major depressive disorder. A meta-analysis and new integration of baseline activation and neural response data. Am J Psychiatry 2012;169:693-703.

66. Scalabrini A, Vai B, Poletti S, et al. All roads lead to the defaultmode network - global source of DMN abnormalities in major depressive disorder. Neuropsychopharmacology 2020;45:2058-69. 